\title{
Histone deacetylase 8 as a novel therapeutic target in oral squamous cell carcinoma
}

\author{
MEE-YOUNG AHN ${ }^{1}$ and JUNG-HOON YOON ${ }^{2}$ \\ ${ }^{1}$ College of Medical and Life Sciences, Division of Bio-industry, Major in Pharmaceutical Engineering, Silla University, \\ Busan 46958; ${ }^{2}$ Department of Oral and Maxillofacial Pathology, College of Dentistry, Wonkwang Bone Regeneration \\ Research Institute, Daejeon Dental Hospital, Wonkwang University, Daejeon 302-120, Republic of Korea
}

Received July 14, 2016; Accepted November 21, 2016

DOI: $10.3892 /$ or.2016.5280

\begin{abstract}
The overexpression of histone deacetylases (HDACs) has been observed in many cancers and inhibition of specific HDAC has emerged as a new target for cancer therapy. The present study examined the expression of HDAC 8 and the inhibitory effect of HDAC8 in oral squamous cell carcinoma (OSCC). The expression of HDAC8 was measured in human OSCC tissues and OSCC cell lines using immunohistochemistry and immunoblotting. HDAC8 was knocked down in OSCC cells by transfection with HDAC8 siRNAs and cell proliferation was quantified. Apoptosis and autophagy were measured using flow cytometry and immunoblotting. HDAC8 were overexpressed in OSCC tissues and OSCC cells, mainly localized in the cytoplasm. HDAC8 siRNAs effectively reduced the level of HDAC8 expression and HDAC8 silencing significantly inhibited the proliferation of OSCC cells. HDAC8 knockdown induced apoptotic cell death through caspases activation and pro-survival autophagy in OSCC cells. Combination with HDAC silencing and autophagy inhibition enhanced cell death by increasing apoptosis in OSCC cells. This study suggests that inhibition of HDAC8 might become a novel therapeutic strategy for OSCC.
\end{abstract}

\section{Introduction}

Oral cancers are the sixth most common malignancies and they affect more than 300,000 new patients being diagnosed every year worldwide $(1,2)$. Of oral cancers $90 \%$ are oral squamous cell carcinoma, the most common malignant neoplasm of the oral cavity $(1,3)$. In fact, in spite of the many advancements made in the field of oral cancer prevention and multimodality

Correspondence to: Professor Jung-Hoon Yoon, Department of Oral and Maxillofacial Pathology, College of Dentistry, Wonkwang Bone Regeneration Research Institute, Daejeon Dental Hospital, Wonkwang University, Dunsan-ro 77, Daejeon 302-120, Republic of Korea

E-mail: opathyoon@wku.ac.kr

Key words: histone deacetylase 8, oral squamous cell carcinoma, HDAC8 siRNA, apoptosis, autophagy treatments, the 5-year survival rate for OSCC remains at a disappointingly stable level, almost unchanged over the past 20 years (4-6). The poor prognosis of OSCC is mainly due to a low response rate to current therapeutic strategies.

Altered molecular expressions might be potential markers for a diagnosis and prognosis of OSCC (7). The susceptibility of an individual to oral cancer is mediated by genetic and environmental factors $(3,8)$. Epigenetics is another major player in multistep carcinogenesis of oral cancers (4). Histone deacetylases (HDACs) play a key role in the epigenetic regulation of genes by catalyzing the removal of acetyl groups. Eighteen HDACs have been characterized in humans and are subdivided into four groups based on their homology to yeast HDACs (9). Class I HDACs are homologous to yeast Rpd3 and consist of HDAC1, 2, 3 and 8 (10). Class II HDACs are homologous to yeast Hda1 and have been subdivided into class IIa (HDAC4, 5, 7 and 9) and IIb (HDAC6 and 10) based on domain organization $(11,12)$. Class III HDACs is composed of the Sirtuins (SIRT) proteins 1-7 and HDAC11 is classified separately as class IV (11). Recently, overexpression of HDACs has been observed in many cancers and inhibition of specific HDAC has emerged as a new target for cancer therapy. Class I HDACs are the most thoroughly investigated with respect to function and relevance for tumor formation and progression (13). HDAC1, HDAC2 and HDAC3 expression were associated with advanced-stage disease and poor prognosis $(14,15)$. High HDAC8 expression was also observed in advanced stage of neuroblastoma (16). Previous studies showed that HDAC2 expression was overexpressed in paraffin-embedded biopsies from OSCC patients. However, the correlation between HDAC8 expression and oral cancer has not been reported. The present study examined the expression of HDAC8 and the inhibitory effect of HDAC8 in OSCC.

\section{Materials and methods}

Cell culture and reagent. The human OSCC YD-8 cells, YD-10B cells and SNU-1076 cells were purchased from the Korea Cell Line Bank (Seoul, Korea). The human OSCC FaDu cells were purchased from the American Type Culture Collection (ATCC; Rockville, MD, USA). YD-8, YD-10B and SNU-1076 cell lines were incubated in RPMI-1640 medium (Gibco, Rockville, MD, USA) containing 10\% fetal bovine 
serum (FBS; Gibco) and $100 \mathrm{U} / \mathrm{ml}$ penicillin-streptomycin (Invitrogen, Carlsbad, CA, USA). FaDu cell lines were incubated in MEM medium (Gibco) containing 10\% FBS (Gibco) and $100 \mathrm{U} / \mathrm{ml}$ penicillin-streptomycin (Invitrogen). Immortalized normal oral keratinocytes (INOK) was used for normal control, as described in a previous study (17) and was incubated in Dulbecco's modified Eagle's medium (DMEM) containing 10\% FBS (Gibco) and $100 \mathrm{U} / \mathrm{ml}$ penicillin-streptomycin (Invitrogen). All cells were maintained as monolayers at $37^{\circ} \mathrm{C}$ in an atmosphere containing $5 \% \mathrm{CO}_{2} /$ air.

Tissue sample preparation. Tissue samples from patients were collected during surgery or biopsy after obtaining informed consents from patients in Wonkwang Dental Hospital. Fourteen OSCC patients confirmed by pathological diagnosis were included in the present study. Median age of these patients was 57 years, 9 patients were male and 5 females. Eight inflammatory gingival inflammatory hyperplasia or fibrous hyperplasia of the buccal mucosa were examined as benign controls. Median age of these patients was 53 years, 4 patients were male and 4 females. Samples were fixed in $10 \%$ buffered formalin and then embedded in paraffin. All surgically resected specimens were sectioned for histopathological examination and immunohistochemical detections. The protocol for the present study was approved by the ethics committee of Wonkwang University, School of Dentistry.

Immunohistochemistry. Paraffin-embedded tissues were used to identify HDAC8 expression. Deparaffinization was achieved with xylene followed by a descending series of ethanol concentrations. Antigen retrieval was carried out in a microwave-heated citrate buffer ( $\mathrm{pH} \mathrm{6.0)}$ for $20 \mathrm{~min}$. The endogenous peroxidases were blocked with $3 \% \mathrm{H}_{2} \mathrm{O}_{2} /$ methanol for $15 \mathrm{~min}$ at room temperature. The non-specific epitopes were blocked with $1 \%$ normal goat serum for $30 \mathrm{~min}$ at room temperature. The tissue sections were incubated overnight at $4^{\circ} \mathrm{C}$ with HDAC8 antibody (1:100; Abcam, Cambridge, MA, USA). The immunoreactions were visualized using a streptavidin-biotin complex method followed by a diaminobenzidine reaction (Invitrogen). The tissue sections were counterstained with hematoxylin in order to visualize the nuclei. The immunoreactions were viewed under an optical microscope (magnification, x400; Leica) and the images recorded on a digital camera (Olympus Optical, Co., Ltd., Tokyo, Japan).

Trypan blue exclusion assay. The trypan blue exclusion assay was based on the capability of viable cells to exclude the dye. Five minutes later $0.4 \%$ trypan blue (Gibco) was added to cells, they were loaded into a hematocytometer and counted for the dye uptake. The number of viable cells was calculated as the percentage of the total cell population.

siRNA transfection. The siRNA oligonucleotides for HDAC8 were purchased from Genolution Pharmaceuticals (Seoul, Korea), and the non-targeting control siRNA (NC. siRNA) was used as the negative control. The human HDAC8 siRNA sequences are as follows: HDAC8 siRNA \#1, sense 5'-CGAG UAUGUCAGUAUGUGU(UU)-3' and antisense 5'-ACACAU ACUGACAUACUCG(UU)-3'; HDAC8-siRNA \#2, sense
5'-CAUAUGCACUGCAUAAGCA(UU)-3' and antisense 5'-UGCUUAUGCAGUGCAUAUG(UU)-3'. The cells were transfected with $100 \mathrm{nM}$ siRNA for $24 \mathrm{~h}$ using Lipofectamine RNAiMAX reagent (Invitrogen). The cells were harvested for protein analysis after $48 \mathrm{~h}$. Western blot analysis was used to validate the silencing of protein expression.

Western blot analysis. The cells were washed with phosphatebuffered saline (PBS) and harvested in lysis buffer. Samples containing equal amounts of protein were resolved on SDS-polyacrylamide gel in a 6-15\% gel, transferred to a polyvinylidene difluoride (PVDF) membrane (NEN Life Science, Inc., Boston, USA), and probed sequentially with antibodies against HDAC8 (Millipore, Bedford, MA, USA), cleaved caspase-9, procaspase-7, procaspase-3, PARP, LC3B, Beclin-1, ATG5, ATG12, p62 (Cell Signaling Technology, Danvers, MA, USA) and actin (Santa Cruz Biotechnology, Santa Cruz, CA, USA). The blots were developed using an enhanced chemiluminescence (ECL) kit (Amersham, Cardiff, UK).

Apoptosis analysis by flow cytometry. The cells were fixed in chilled $75 \%$ methanol and stained with a propidium iodine (PI) solution (100 $\mu \mathrm{g} / \mathrm{ml}$ RNase and $10 \mu \mathrm{g} / \mathrm{ml}$ PI in PBS) for cell cycle analysis. The cells were stained to the Vybrant ${ }^{\circledR}$ apoptosis assay kit (Molecular Probes, Eugene, OR, USA), followed by labeling Alexa Fluor ${ }^{\circledR} 488$ Annexin V and PI for apoptosis analysis. Data acquisition and analysis was carried out using Cell Llab Quanta ${ }^{\mathrm{TM}}$ SC flow cytometry (Beckman Coulter, Inc., Miami, FL, USA) and software.

Detection of acidic vesicular organelles (AVOs). Autophagy is characterized by the formation and promotion of AVO. To detect the development of AVOs, the cells were stained with acridine orange as previously described (18). In acridine orange stained cells, the cells fluoresce bright green, whereas acidic compartments fluoresce bright red. Briefly, the treated cells were stained with acridine orange $(1 \mu \mathrm{g} / \mathrm{ml})$ for $15 \mathrm{~min}$. To quantify the development of AVOs, the stained cells were analyzed using FACScan flow cytometer and CellQuest software (Beckman Coulter, Inc.).

Statistical analysis. Data are expressed as the mean \pm SEM of at least three individual experiments. Statistical comparisons between groups were performed using two-tailed Student's t-test (Microsoft Excel). Statistical significance was set at ${ }^{*} \mathrm{P}<0.05,{ }^{* *} \mathrm{P}<0.01$ and ${ }^{* * *} \mathrm{P}<0.001$.

\section{Results}

Expression level of HDAC8 in OSCC tissues and OSCC cell lines. To identify whether HDAC8 is highly expressed in OSCC, we examined the expression of HDAC8 in benign controls and tumor tissues using immunohistochemstry. As shown in Fig. 1A, the level of HDAC8 expression was markedly overexpressed in OSCC tissues compared to benign control tissues. HDAC8 expression was mainly observed in the cytoplasm of tissues, especially epithelial cells. To confirm overexpression of HDAC8 in OSCC, we compared the levels of HDAC8 expression between several human OSCC cell lines and INOK cells using western blot analysis. Overexpression of 
A

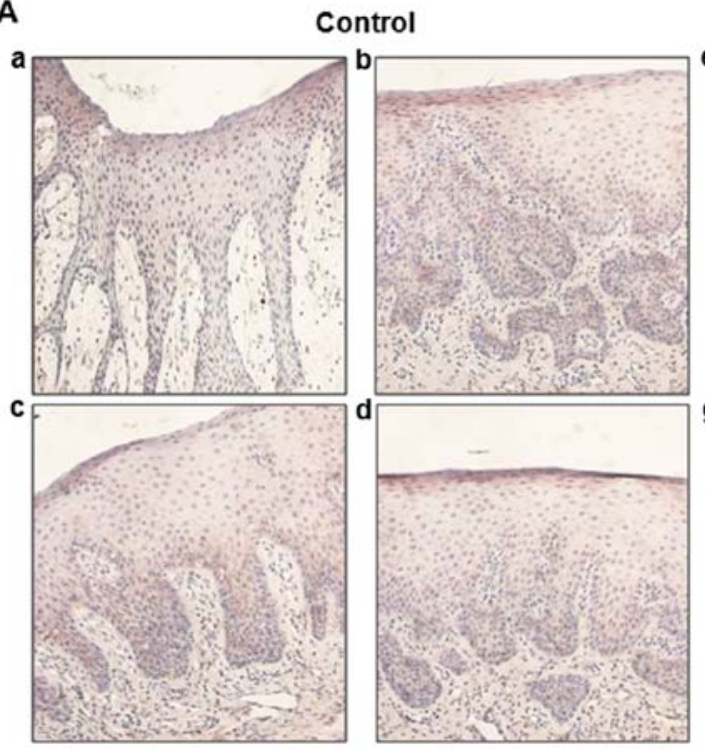

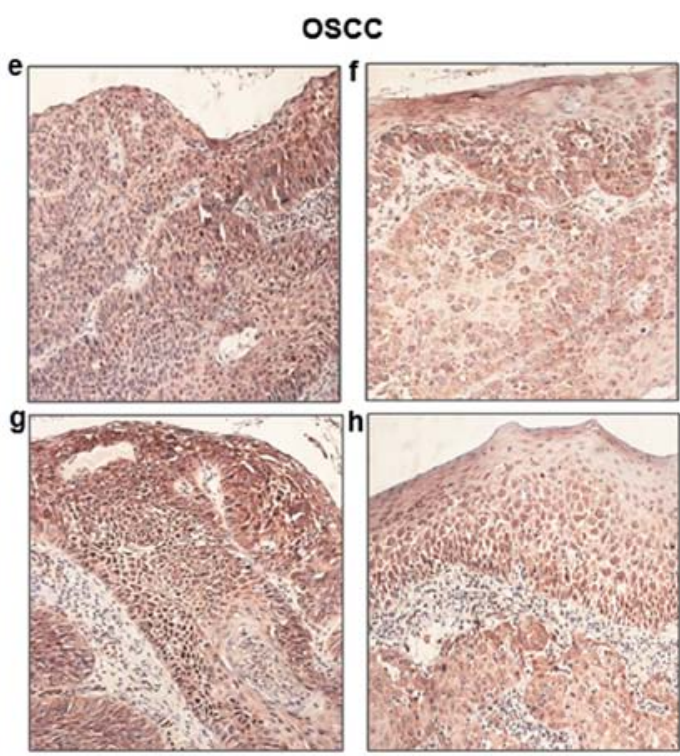

B

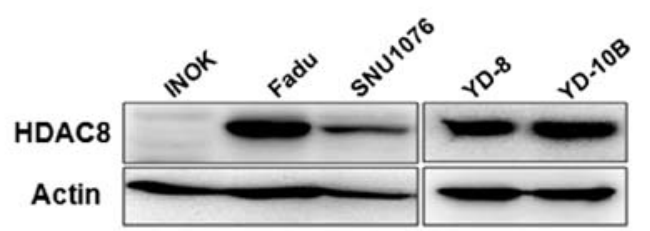

Figure 1. Expression of HDAC8 in OSCC. (A) Immunohistochemistry by HDAC8 antibody in human OSCC tissues. (a-d) benign control tissues; (e-h) OSCC tissues. The images were taken at magnification, x200. (B) Western blotting by HDAC8 antibody in INOK and OSCC cell lines. The amount of protein was normalized to levels of actin. The representative bands from three independent experiments are shown.

HDAC8 were observed in several OSCC cell lines, whereas there was no expression in INOK cells (Fig. 1B). Especially, HDAC8 was highly expressed in YD-10B and FaDu cells than other cell lines. Therefore, YD-10B and FaDu cell lines were selected for the subsequent experiments.

HDAC8 knockdown inhibits the OSCC cell proliferation. To determine whether the expression of HDAC8 is associated with cell proliferation in OSCC, HDAC8 was knocked down in YD-10B and FaDu cells by transfection of HDAC8 siRNAs. YD-10B and FaDu cells were transfected with two different siRNAs specific for HDAC8 and the knockdown effect of HDAC8 siRNAs was determined by western blotting. Transfection with HDAC8 siRNAs reduced targeted HDAC8 expression in both YD-10B and FaDu cells, whereas the nonspecific targeting control did not affect HDAC8 expression (Fig. 2A). As shown in Fig. 2B, HDAC8 silencing significantly inhibited the proliferation of OSCC cells by both HDAC8 siRNA transfections. HDAC8 siRNA\#2 was shown to more efficiently silence HDAC8 expression and inhibit cell proliferation than HDAC8 siRNA\#1 in both OSCC cells. Therefore, HDAC8 siRNA \#2 was used for further HDAC8 knockdown study. Furthermore, HDAC8 siRNA\#2 supressed cell proliferation in a time-dependent manner (Fig. 2C).

HDAC8 silencing induces apoptotic cell death. To assess whether the growth inhibitory effects by HDAC8 knockdown were associated with the induction of apoptosis, we first examined Annexin V/PI staining by flow cytometry assay. As shown in Fig. 3A, HDAC8 knockdown by HDAC8 siRNA transfection significantly increased the number of Annexin V positive apoptotic cells compared with the NC siRNA transfected control. The expression of apoptosis-related proteins was next measured to determine the mechanism of apoptosis using western blot analysis. The transfection with HDAC8 siRNA markedly induced the decrease of procaspase-3, -7 and the increase of cleaved caspase- 9 in OSCC cells. HDAC8 knockdown also increased the level of PARP cleavage, a known endogenous substrate for caspases (Fig. 3B). These data indicated that HDAC8 knockdown induced apoptosis by activating caspases, which cleaved PARP in OSCC cells.

Autophagy induction by HDAC8 silencing. We next determined whether HDAC8 silencing can induce autophagy in OSCC cells. The levels of autophagy-related gene expression were examined in HDAC8 siRNA trasfected OSCC cells. As shown in Fig. 4A, the transfection with HDAC8 siRNA markedly increased the levels of Beclin-1, ATG5 and ATG12 which are involved in the early stage of autophagosome formation. The level of the microtubule-associated protein 1 light change 3 (LC3)-II, a marker for autophagic vesicles was also increased in HDAC8-silenced cells compared with control cells. In addition, reduction of p62 as a marker for autophagic flux was observed in HDAC8 siRNA transfected cells. The autophagy response to HDAC8 silencing was next confirmed by acridine orange staining with flow cytometry assay. Acridine orange staining showed that HDAC8 knockdown significantly increased the number of AVOs, redpositive cells, compared to control. Indicated cell percentage over the line showed acidic red positive cell ratios (Fig. 4B). 
A
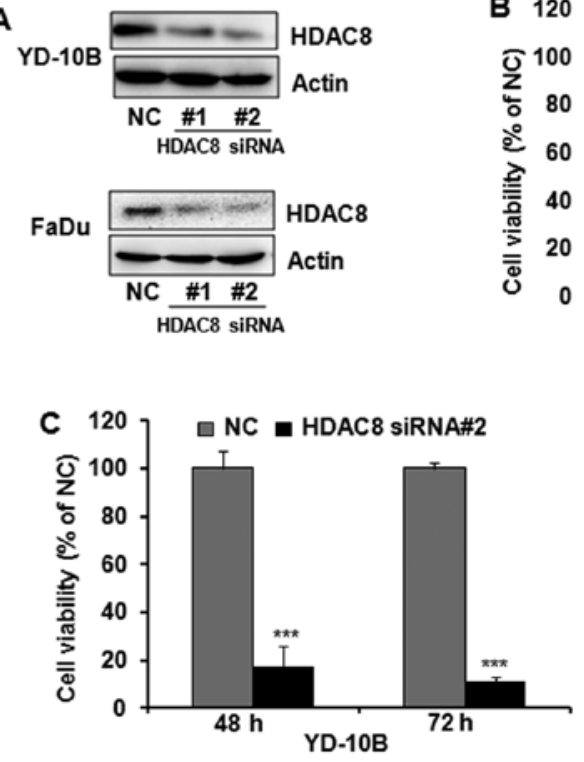
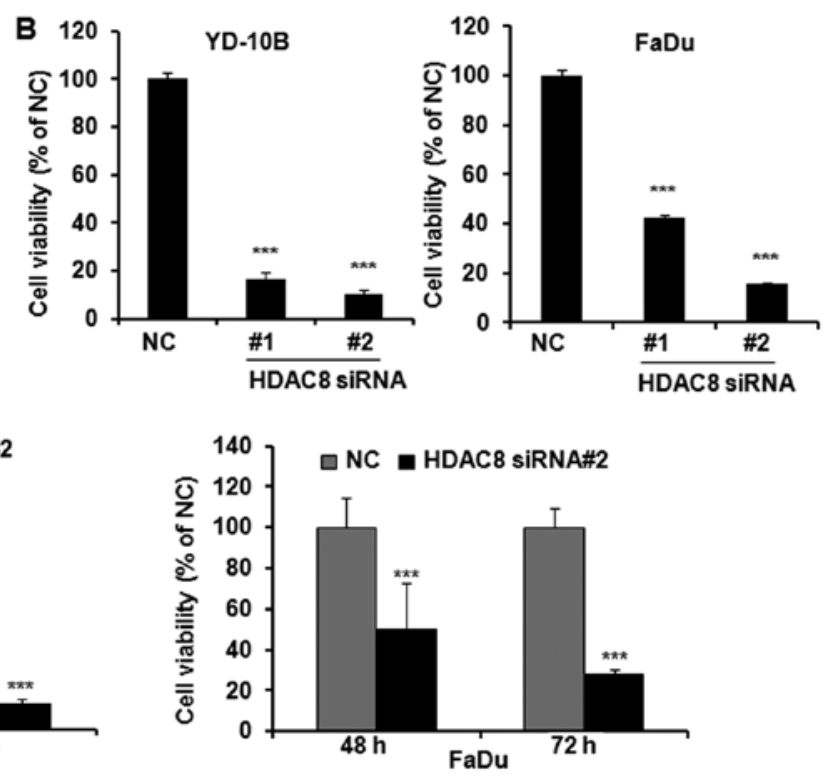

Figure 2. HDAC8 silencing on the proliferation in YD-10B and FaDu cells. (A) Immunoblot analysis of HDAC8 expression in HDAC8 siRNA tranfected YD-10B and FaDu cells. Cells were transfected with HDAC8 siRNA for $24 \mathrm{~h}$ and harvested after $48 \mathrm{~h}$. The total cell lysates were prepared and $30 \mu \mathrm{g}$ of the protein was subjected to SDS-PAGE followed by western blot analysis and chemiluminescent detection. Western blot analysis was performed with HDAC8 antibody. The protein levels were normalized by a comparison to the actin levels. The representative bands from three independent experiments are shown. (B) Cell viability of HDAC8 silenced YD-10B and FaDu cells. Cells were transfected with HDAC8 siRNA for $24 \mathrm{~h}$ and harvested after $48 \mathrm{~h}$. Cell viability was determined using trypan blue exclusion assay after siRNA trasfection. The percentage of viable cells was calculated as the ratio of HDAC 8 siRNA transfected cells to the NC. siRNA transfected control cells. The data are reported as the mean \pm SD of three independent experiments. ${ }^{* * *} \mathrm{P}<0.001$ compared to untreated control. (C) Time-dependent cell viability of HDAC8 silenced YD-10B and FaDu cells. Cells were transfected with HDAC 8 siRNA \#2 for 24 h and harvested after 24 and $48 \mathrm{~h}$. Cell viability was determined using trypan blue exclusion assay after siRNA trasfection. The percentage of viable cells was calculated as the ratio of HDAC8 siRNA transfected cells to the NC. siRNA transfected control cells. NC. siRNA, non-targeting control siRNA. The data are reported as the mean $\pm \mathrm{SD}$ of three independent experiments. ${ }^{* * *} \mathrm{P}<0.001$ compared to untreated control.

A
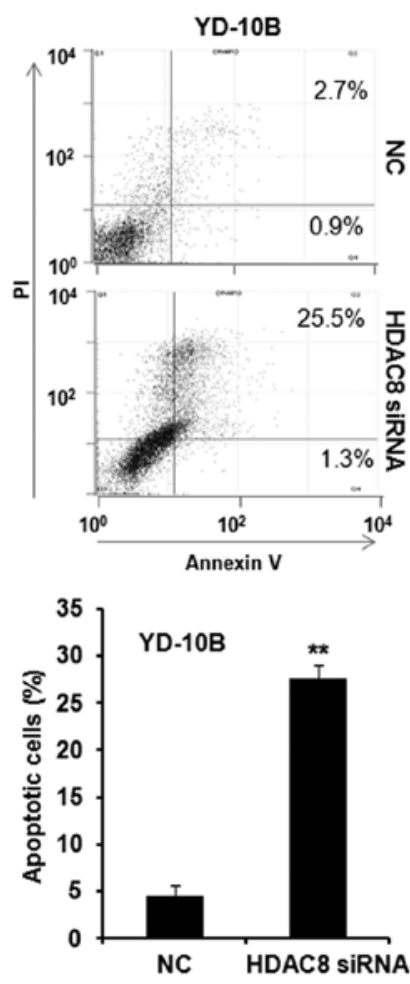

FaDu
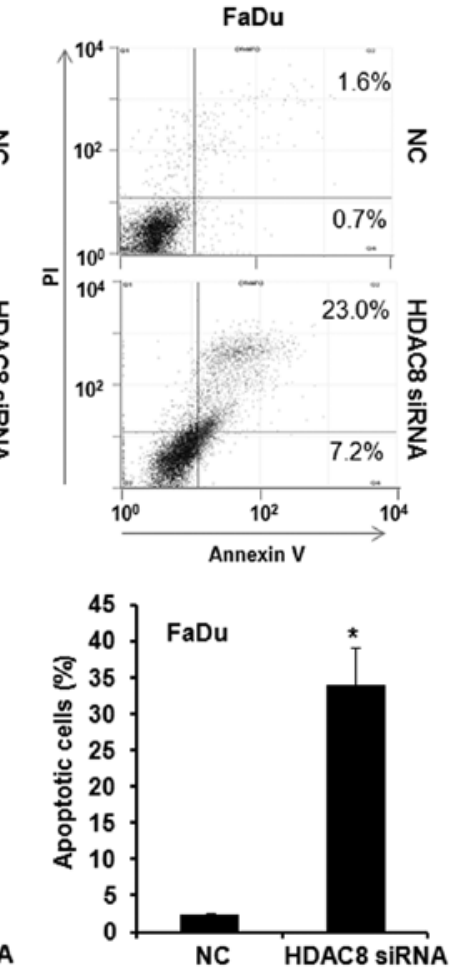

B
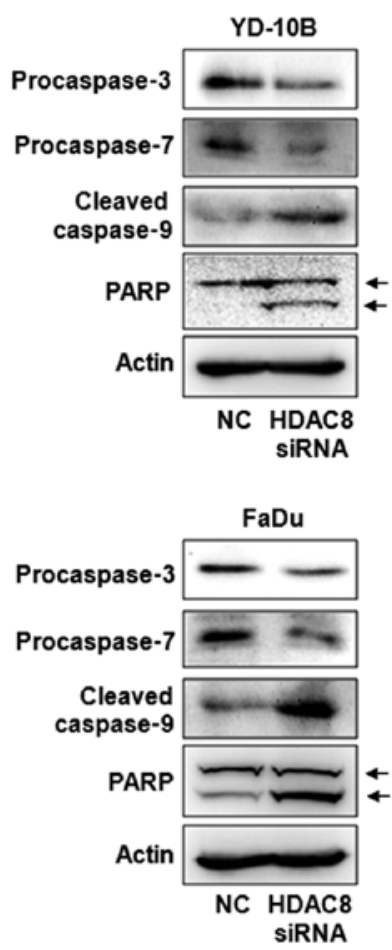

Figure 3. HDAC8 siRNA transfection induces apoptosis in YD-10B and FaDu cells. Cells were transfected with HDAC8 siRNA for $24 \mathrm{~h}$ and harvested after $48 \mathrm{~h}$. (A) Annexin V/PI staining for apoptotic cells. The apoptotic cells were detected using ApopTag In Situ Apoptosis Detection kits and the flow cytometry results are shown. The data are reported as the mean $\pm \mathrm{SD}$ of three independent experiments. ${ }^{*} \mathrm{P}<0.05,{ }^{, * *} \mathrm{P}<0.01$ compared to the NC siRNA transfected control. (B) Expression of the apoptosis-related proteins in YD-10B and FaDu cells. The total cell lysates were prepared and the protein was subjected to SDS-PAGE followed by western blot analysis and chemiluminescent detection. Western blot analysis was performed using a series of antibodies; procaspase-3, procaspase-7, cleaved caspase-9 and PARP. NC. siRNA, non-targeting control siRNA. The protein levels were normalized by a comparison with the actin levels. The representative bands from three independent experiments are shown. 
A
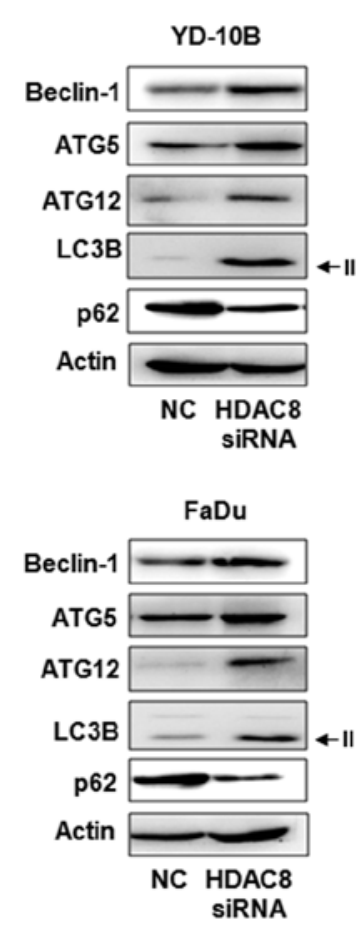

B

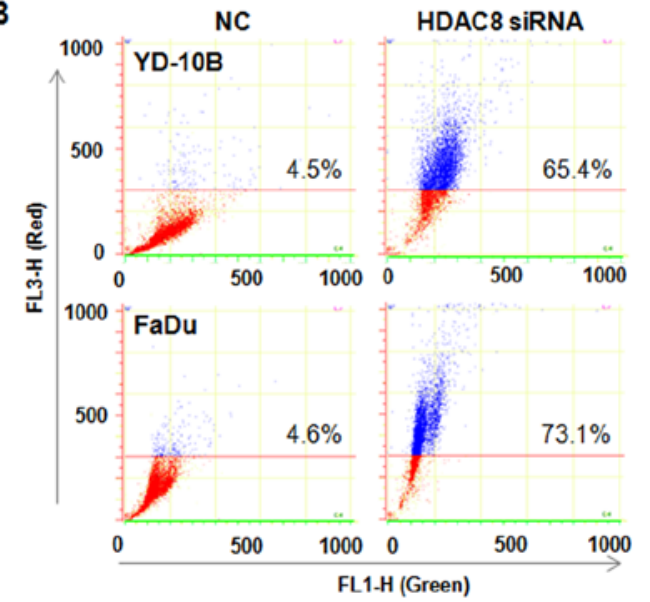

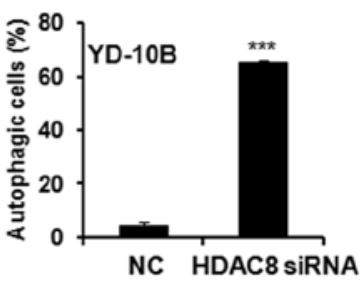

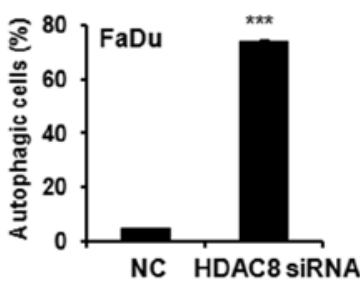

C
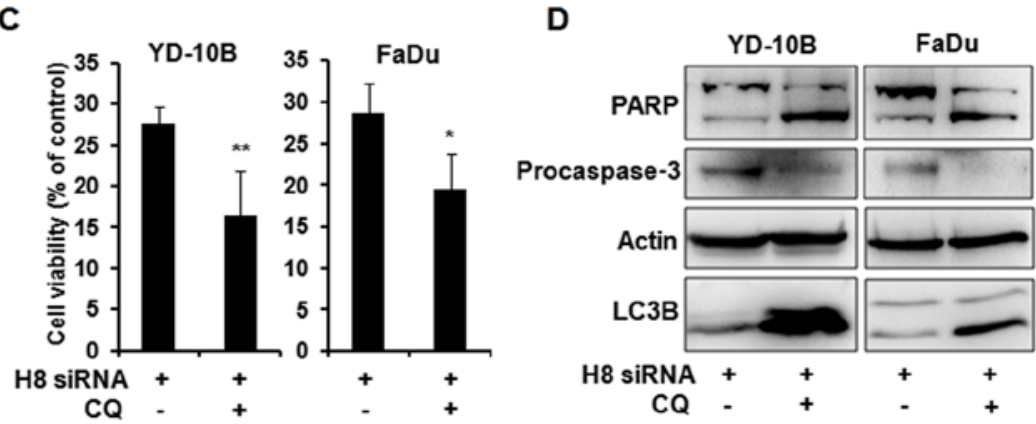

Figure 4. Autophagy in HDAC8 silencing YD-10B and FaDu cells. Cells were transfected with HDAC8 siRNA for $24 \mathrm{~h}$ and harvested after $48 \mathrm{~h}$. (A) Expression of the autophagy-related proteins in YD-10B and FaDu cells. Cell lysates were subjected to western blot analysis using a series of antibodies against Beclin-1, ATG5, ATG12, LC3B and p62. The protein levels were normalized by a comparison to the actin levels. The representative bands from three independent experiments are shown. (B) Fluorescence-activated cell sorting analysis using acridine orange. The cells were stained with acridine orange $(1 \mu \mathrm{g} / \mathrm{ml})$ and then subjected to flow cytometric analysis. FL1-H, green color intensity; FL3-H, red color intensity. Indicated cell percentage over the line show acidic red positive cell ratios. The data are reported as the mean $\pm \mathrm{SD}$ of three independent experiments. ${ }^{* * *} \mathrm{P}<0.001$ compared to the NC siRNA transfected control. (C) The cells were transfected with HDAC 8 siRNA for $24 \mathrm{~h}$ and were harvested after $48 \mathrm{~h}$ incubation with or without chloroquine (CQ, $50 \mu \mathrm{mol} / \mathrm{l})$. Cell viability was determined by trypan blue exclusion assay. The representative results from three independent experiments are shown. ${ }^{*} \mathrm{P}<0.05$, ${ }^{* *} \mathrm{P}<0.01$ compared to the HDAC8 siRNA transfected cells. (D) Western blot analysis of PARP, procaspase-3 and LC3B expressions in HDAC8 siRNA transfected cells with or without CQ $(50 \mu \mathrm{mol} / 1)$. H8 siRNA, HDAC8 siRNA; NC. siRNA, non-targeting control siRNA. The protein levels were normalized by a comparison to the actin levels. The representative bands from three independent experiments are shown.

To evaluate the function of autophagy by HDAC8 silencing, specific autophagy inhibitor chloroquine (CQ) was co-treated with HDAC8 siRNA in OSCC cells. We examined the cell viability using trypan blue exclusion assay. Combined treatment with HDAC8 knockdown and CQ significantly reduced cell viability as compared to HDAC8 knockdown without CQ in both OSCC cells (Fig. 4C). The expression levels of PARP, caspase-3 and LC3B-II were next examined using western blot analysis. The accumulation of LC3B-II by CQ was observed in combined treatment compared to HDAC8 siRNA transfection alone as expected. Furthermore, the level of PARP cleavage was markedly increased and procaspase- 3 expression was decreased in combined treatment with HDAC8 knockdown and CQ compared to HDAC8 knockdown alone, these results indicated that inhibition of autophagy enhanced HDAC8 silencing-induced apoptosis (Fig. 4D).

\section{Discussion}

Class I HDACs have been most widely studied in their classical role as histone modifiers and transcriptional repressors (19). The most frequently studied and best-characterized human HDACs are HDAC1 and HDAC2. Also, HDAC8 is most recently identified class I HDAC and the role of HDAC8 in normal and cancer cells remains unclear $(20,21)$. The expression of HDAC 8 has been determined in several cancer tissues. Upregulation of HDAC8 was detected in colon, urothelial, ovarian and endometrial cancers $(13,20,22,23)$, and high HDAC8 expression was associated with markers of poor prognosis and poor overall survival in neuroblastoma (16). Likewise, our results also showed that HDAC8 were markedly upregulated in both OSCC tissues and OSCC cell lines. However, there was no expression in INOK cells and weak expression in benign control tissues. This study first determined the expression of HDAC8 in OSCC, therefore, HDAC8 upregulation can be a diagnostic marker for OSCC and may be associated with oral carcinogenesis.

HDACs were first identified as enzymes that function to remove acetyl groups from histones $(24,25)$. To exert their function, HDACs need to be in the nucleus (26). However, recent studies suggest that the primary substrates of HDAC enzymes are not histone proteins, but non-histone proteins $(24,27)$. It could be demonstrated that the subcellular localization of HDAC can be nuclear as well as cytoplasmic. The class I HDACs are found primarily in the nucleus of most cell types and form nuclear multiprotein complexes that 
interact with other chromatin modifiers and transcription factors $(10,20)$. Unlike other class I HDACs, the subcellular localization of HDAC8 seems to vary with cell types $(20,28)$. Nakagawa et al (22) reported that HDAC8 expression was detected in both the nucleus and the cytoplasm for gastric adenocarcinoma, esophageal squamous cell carcinoma, prostate carcinoma and breast papillotubular carcinoma, whereas nuclear localization of HDAC8 was detected in non-cancerous gastric, esophageal or prostate epithelium. However, cytosolic expression of HDAC8 was described in differentiating smooth muscle cells and prostate stromal cells $(29,30)$. In this study, the immunohistochemistry results showed that overexpressed HDAC8 was mainly distributed in the cytoplasm of OSCC tissues. Although the expression levels of HDAC8 were very weak in benign control tissues, the localization of HDAC8 was also observed in the cytoplasm. HDAC localization is connected to modulate key cellular processes, including transcriptional regulatory function (31). Previous studies reported that HDAC8 knockdown did not affect global histone H4 acetylation $(16,20)$, but induced cytoplasm protein $\alpha$-tubulin acetylation $(20,32)$, which provided a strong indication for the cytosolic localization of HDAC8. The results indicated that HDAC8 is localized in the cytoplasm of oral tissues and, therefore, we carefully suggest that it may be associated with regulating the non-histone protein response, rather than histone deactylation function. Further study will be needed to investigate the correlation of HDAC8 localization and its function in OSCC.

HDAC appears to be important in the regulation of proliferation in cancer cells. However, the biological function of specific HDACs is still unknown. Although the present study showed upregulation of HDAC8 in OSCC, it is not sure that HDAC8 inhibition might be a suitable therapeutic target for OSCC. Therefore, we next examined whether siRNA-mediated HDAC8 knockdown induce cell growth inhibition in OSCC cells. The results showed that HDAC8 siRNA markedly reduced the levels of HDAC8 and HDAC8 knockdown significantly inhibited the cell proliferation in both OSCC cell lines. Although there was the same effect of HDAC8 knockdown-induced cell growth inhibition in several cancer cells, the apoptosis induction by HDAC8 inhibition was different. HDAC8 inhibition by pharmacological drug or siRNA induced apoptosis in human and murine-derived maligant peripheral nerve sheath tumors and hepatocellular carcinoma, whereas there was a limited induction of apoptosis after either HDAC8 knockdown or pharmacological inhibitor treatment in urothelical cancer cell lines $(20,21,33)$. Consistent with the former findings, the present study showed that HDAC8 knockdown markedly induced apoptosis through caspases activation in OSCC cells. The results implied that HDAC8 might play an important role in regulating cell proliferation and apoptosis in OSCC cells.

Apoptosis plays a major controling role in cancer cell death, but recent reports have demonstrated that autophagy is also an important molecular mechanism for cancer cell death $(34,35)$. While autophagy is constitutively important for intracellular quality control and maintenance of cellular homeostasis from the cytoplasm to lysosomes, autophagy is frequently activated to much higher levels in cancer cells in response to a variety of chemotherapeutic treatments (36-39). In recent studies, HDAC6 is dispensable for starvation-induced autophagy and HDAC8 is degraded via autophagy and the ubiqitin-proteasesome system in lung cancer cells $(40,41)$. We previously determined that HDAC inhibitor apicidin induced both apoptosis and autophagy in OSCC cells (42), but the correlation between autophagy induction and specific HDAC inhibition has not been reported in cancer cells. In the present results, HDAC8 silencing markedly increased the levels of major autophagic related proteins and autophagic vesicles in OSCC cells. In addition, autophagy inhibition by CQ increased apoptotic cell death in HDAC8 siRNA transfected OSCC cells, which means autophagy by HDAC8 knockdown have pro-survival effect in OSCC cells. These results are similar to our previous study for HDAC inhibitor apicidin-treated OSCC cells (42). We wondered whether HDAC8 is decreased in apicidin-treated OSCC cells. The expression of HDAC8 was also markedly inhibited in apicidin treated OSCC cells (data not shown). Overall, HDAC8 inhibition by siRNA or pharmacological drug may be highly related with autophagy induction as a pro-survival function and inhibitors of autophagy could increase the antitumor effect of HDAC8 inhibition in OSCC cells.

In conclusion, HDAC8 overexpressed in OSCC tissues and OSCC cell lines are mainly localized in the cytoplasm. HDAC8 knockdown inhibited cancer cell proliferation and activated both caspase-dependent apoptotic cell death and pro-survival autophagy in OSCC cells. Moreover, combined treatment with HDAC8 knockdown and autophagy inhibitor enhanced cell death through apoptosis induction. Taken together, these findings provide new and important information on the diagnosis of OSCC and HDAC8 could be an effective molecular target of antitumor therapy for patients with OSCC.

\section{Acknowledgements}

The present study was supported by the Basic Science Research Program through the National Research Foundation of Korea (NRF) funded by the Ministry of Education, Science and Technology (NRF-2011-0023907, NRF-2016R1D1A3B03931034).

\section{References}

1. Effiom OA, Adeyemo WL, Omitola OG, Ajayi OF, Emmanuel MM and Gbotolorun OM: Oral squamous cell carcinoma: a clinicopathologic review of 233 cases in Lagos, Nigeria. J Oral Maxillofac Surg 66: 1595-1599, 2008.

2. Zushi Y, Narisawa-Saito M, Noguchi K, Yoshimatsu Y, Yugawa T, Egawa N, Fujita M, Urade M and Kiyono T: An in vitro multistep carcinogenesis model for both HPV-positive and -negative human oral squamous cell carcinomas. Am J Cancer Res 1: 869-881, 2011.

3. Weng CJ, Hsieh YH, Chen MK, Tsai CM, Lin CW and Yang SF: Survivin SNP-carcinogen interactions in oral cancer. J Dent Res 91: 358-363, 2012.

4. Mascolo M, Siano M, Ilardi G, Russo D, Merolla F, De Rosa G and Staibano S: Epigenetic disregulation in oral cancer. Int J Mol Sci 13: 2331-2353, 2012.

5. Mydlarz WK, Hennessey PT and Califano JA: Advances and perspectives in the molecular diagnosis of head and neck cancer. Expert Opin Med Diagn 4: 53-65, 2010.

6. Jemal A, Siegel R, Ward E, Hao Y, Xu J, Murray T and Thun MJ: Cancer statistics, 2008. CA Cancer J Clin 58: 71-96, 2008.

7. Chen YJ, Chang JT, Liao CT, Wang HM, Yen TC, Chiu CC, $\mathrm{Lu} \mathrm{YC,} \mathrm{Li} \mathrm{HF} \mathrm{and} \mathrm{Cheng} \mathrm{AJ:} \mathrm{Head} \mathrm{and} \mathrm{neck} \mathrm{cancer} \mathrm{in} \mathrm{the} \mathrm{betel}$ quid chewing area: recent advances in molecular carcinogenesis. Cancer Sci 99: 1507-1514, 2008. 
8. Agrawal D, Gupta S, Agarwal D, Gupta OP and Agarwal M Role of GSTM1 and GSTT1 polymorphism: susceptibility to oral submucous fibrosis in the North Indian population. Oncology 79: 181-186, 2010

9. Marks PA and Dokmanovic M: Histone deacetylase inhibitors: discovery and development as anticancer agents. Expert Opin Investig Drugs 14: 1497-1511, 2005.

10. Delcuve GP, Khan DH and Davie JR: Targeting class I histone deacetylases in cancer therapy. Expert Opin Ther Targets 17: 29-41, 2013.

11. Lucio-Eterovic AK, Cortez MA, Valera ET, Motta FJ, Queiroz RG, Machado HR, et al: Differential expression of 12 histone deacetylase (HDAC) genes in astrocytomas and normal brain tissue: class II and IV are hypoexpressed in glioblastomas. BMC Cancer 8: 243-253, 2008.

12. Martin M, Kettmann R and Dequiedt F: Class IIa histone deacetylases: regulating the regulators. Oncogene 26: 54505467,2007

13. Weichert W, Denkert C, Noske A, Darb-Esfahani S, Dietel M Kalloger SE, Huntsman DG and Köbel M: Expression of Class histone deacetylases indicates poor prognosis in endometrioid subtypes of ovarian and endometrial carcinomas. Neoplasia 10: 1021-1027, 2008

14. Iglesias-Linares A, Yañez-Vico RM and González-Moles MA: Potential role of HDAC inhibitors in cancer therapy: insights into oral squamous cell carcinoma. Oral Oncol 46: 323-329, 2010.

15. Chang HH, Chiang CP, Hung HC, Lin CY, Deng YT and Kuo MY: Histone deacetylase 2 expression predicts poorer prognosis in oral cancer patients. Oral Oncol 45: 610-614, 2009.

16. Oehme I, Deubzer HE, Wegener D, Pickert D, Linke JP, Hero B, Kopp-Schneider A, Westermann F, Ulrich SM, von Deimling A, et al: Histone deacetylase 8 in neuroblastoma tumorigenesis. Clin Cancer Res 15: 91-99, 2009.

17. Kim SA, Kwon SM, Yoon JH and Ahn SG: The antitumor effect of PLK1 and HSF1 double knockdown on human oral carcinoma cells. Int J Oncol 36: 867-872, 2010.

18. Kanematsu S, Uehara N, Miki H, Yoshizawa K, Kawanaka A, Yuri $\mathrm{T}$ and Tsubura A: Autophagy inhibition enhances sulforaphane-induced apoptosis in human breast cancer cells. Anticancer Res 30: 3381-3390, 2010.

19. Shakespear MR, Halili MA, Irvine KM, Fairlie DP and Sweet MJ: Histone deacetylases as regulators of inflammation and immunity. Trends Immunol 32: 335-343, 2011.

20. Lehmann M, Hoffmann MJ, Koch A, Ulrich SM, Schulz WA and Niegisch G: Histone deacetylase 8 is deregulated in urothelial cancer but not a target for efficient treatment. J Exp Clin Cancer Res 10: 59, 2014.

21. Lopez G, Bill KL, Bid HK, Braggio D, Constantino D, Prudner B, Zewdu A, Batte K, Lev D and Pollock RE: HDAC8, a potential therapeutic target for the treatment of malignant peripheral nerve sheath tumors (MPNST). PLoS One 10: e0133302, 2015.

22. Nakagawa M, Oda Y, Eguchi T, Aishima S, Yao T, Hosoi F, Basaki Y, Ono M, Kuwano M, Tanaka M, et al: Expression profile of class I histone deacetylases in human cancer tissues. Oncol Rep 18: 769-774, 2007.

23. Niegisch G, Knievel J, Koch A, Hader C, Fischer U, Albers P and Schulz WA: Changes in histone deacetylase (HDAC) expression patterns and activity of HDAC inhibitors in urothelial cancers. Urol Oncol 31: 1770-1779, 2013.

24. Kim HJ and Bae SC: Histone deacetylase inhibitors: molecular mechanisms of action and clinical trials as anti-cancer drugs. Am J Transl Res 3: 166-179, 2011.
25. Gray SG and Ekstrom TJ: The human histone deacetylase family. Exp Cell Res 262: 75-83, 2001.

26. de Ruijter AJ, van Gennip AH, Caron HN, Kemp S and van Kuilenburg AB: Histone deacetylases (HDACs): characterization of the classical HDAC family. Biochem J 370: 737-749, 2003.

27. Gregoretti IV, Lee YM and Goodson HV: Molecular evolution of the histone deacetylase family: functional implications of phylogenetic analysis. J Mol Biol 338: 17-31, 2004.

28. Oehme I, Deubzer HE, Lodrini M, Milde T and Witt O: Targeting of HDAC8 and investigational inhibitors in neuroblastoma. Expert Opin Investig Drugs 18: 1605-1617, 2009.

29. Waltregny D, De Leval L, Glénisson W, Ly Tran S, North BJ, Bellahcène A, Weidle U, Verdin E and Castronovo V: Expression of histone deacetylase 8 , a class I histone deacetylase, is restricted to cells showing smooth muscle differentiation in normal human tissues. Am J Pathol 165: 553-564, 2004.

30. Waltregny D, North B, Van Mellaert F, de Leval J, Verdin E and Castronovo V: Screening of histone deacetylases (HDAC) expression in human prostate cancer reveals distinct class I HDAC profiles between epithelial and stromal cells. Eur J Histochem 48: 273-290, 2004.

31. Mathias RA, Guise AJ and Cristea IM: Post-translational modifications regulate class IIa histone deacetylase (HDAC) function in health and disease. Mol Cell Proteomics 14: 456-470, 2015.

32. Quan P, Moinfar F, Kufferath I, Absenger M, Kueznik T, Denk H, Zatloukal K and Haybaeck J: Effects of targeting endometrial stromal sarcoma cells via histone deacetylase and PI3K/AKT/ mTOR signaling. Anticancer Res 34: 2883-2897, 2014.

33. Wu J, Du C, Lv Z, Ding C, Cheng J, Xie H, Zhou L and Zheng S: The up-regulation of histone deacetylase 8 promotes proliferation and inhibits apoptosis in hepatocellular carcinoma. Dig Dis Sci 58: 3545-3553, 2013

34. Liu JJ, Lin M, Yu JY, Liu B and Bao JK: Targeting apoptotic and autophagic pathways for cancer therapeutics. Cancer Lett 300: 105-114, 2011.

35. Hannigan AM and Gorski SM: Macroautophagy: the key ingredient to a healthy diet? Autophagy 5: 140-151, 2009.

36. Zhang Q, Yang W, Man N, Zheng F, Shen Y, Sun K, Li Y and Wen LP: Autophagy-mediated chemosensitization in cancer cells by fullerene C60 nanocrystal. Autophagy 5: 1107-1117, 2009.

37. Levine B: Cell biology: Autophagy and cancer. Nature 446: 745-747, 2007.

38. Kondo Y, Kanzawa T, Sawaya R and Kondo S: The role of autophagy in cancer development and response to therapy. Nat Rev Cancer 5: 726-734, 2005.

39. Jin SK and White E: Tumor suppression by autophagy through the management of metabolic stress. Autophagy 4: 563-566, 2008.

40. Lee JY and Yao TP: Quality control autophagy: a joint effort of ubiquitin, protein deacetylase and actin cytoskeleton. Autophagy 6: 555-557, 2010.

41. Park JY and Juhnn YS: cAMP signaling increases histone deacetylase 8 expression by inhibiting JNK-dependent degradation via autophagy and the proteasome system in H1299 lung cancer cells. Biochem Biophys Res Commun 470: 336-342, 2016.

42. Ahn MY, Ahn SG and Yoon JH: Apicidin, a histone deaceylase inhibitor, induces both apoptosis and autophagy in human oral squamous carcinoma cells. Oral Oncol 47: 1032-1038, 2011. 